\title{
Investigation of fabric evolution using bidirectional shear wave velocity measurements
}

\author{
Kazem Fakharian ${ }^{1, ~ *}$, Farzad Kaviani Hamedani ${ }^{1}$, Iman Parandian ${ }^{1}$, Morteza Jabbarpour Aghdam ${ }^{1}$ \\ ${ }^{1}$ Amirkabir University of Technology, Department of Civil \& Environmental Engineering, Tehran, Iran
}

\begin{abstract}
In order to characterise fabric evolution, continuous bidirectional shear wave velocity measurements are performed in vertical and horizontal directions $(\mathrm{V} \& \mathrm{H})$ on triaxial soil specimens during shearing in which two horizontal piezo-electrics were mounted on samples using a new measurement technique. The specimens are prepared by wet tamping method and then subjected to strain-controlled compressional shearing under drained and undrained conditions. The shear wave velocities of all drained specimens initially increased as the loading commenced and then converged to a unique state in both horizontal and vertical directions. The shear wave velocity of undrained specimens on the other hand, for both horizontal and vertical directions initially decreased due to the rising of the excess pore water pressure and then gradually approached a unique shear wave velocity like drained specimens. The fabric condition or stiffness in V\&H directions of all the examined drained and undrained specimens at critical state are found to be unique.
\end{abstract}

\section{Introduction}

Various factors such as grading, grain size, grain shape, relative density, grain arrangement, draining conditions, stress path, mean effective stress, and anisotropy can affect the behaviour of sands. However, the fabric of soil as one of the major factors affecting the behaviour of sands is known to a lesser extent. Several studies are available in literature that have marginally touched the subject and this could have been due to the complexities and requirements of specific instruments.

The critical state is defined as the state where under constant stress, the material subjected to shear stress deforms at constant volume. Classical critical state theory assumes that the soil fabric at critical state becomes isotropic (Roscoe et al. 1958 and Schofield and Wroth, 1968) [1,2]. This is while soil behaves anisotropically during shearing due to microscopic characteristics of soils (Nakata et al. 1998 and Yoshimine et al. 1998) [3, 4]. On the other hand, there are strong evidences that fabric becomes greatly anisotropic at critical state (Oda 1972, Masson and Martinez 2001 and Li and Li 2009) [5-7]. So it is possible that soil behaves anisotropically at critical state too. The study of Song and Dafalias showed that the anisotropic fabric and its evolution towards a critical state had been neglected in classical critical state. Li \& Dafalias (2012) and Yan \& Zhang (2013) used DEM numerical modeling showing that the critical state line is unique at very large strains $[8,9]$. It has also been found that the variations of fabric or loading mode cannot change the Critical State Line (CSL), while an additional requirement of critical value for an appropriate measure of fabricanisotropy is needed. Those studies also showed that dense soil specimens which are having intensive dilatancy require undergoing larger strains to achieve critical fabric tensor. Sadrekarimi \& Olson (2011) using ring shear apparatus showed that if extra strains are induced, the required rearrangement and crushing of the grains will occur simultaneously to reach a unique critical state [10].

On the other hand, stiffness of soil in very small strain $\left(\mathrm{G}_{0}\right)$ can greatly be effected by fabric of soil due to grain arrangements and grain contacts. Values of the shear modulus at very small strains, $\mathrm{G}_{0}$, can be measured using a common laboratory technique that involves transmitting and receiving shear waves using small electro-mechanical transducers known as bender elements (Shirley \& Hampton, 1977) [11]. Continuous monitoring of bender element during triaxial testing were performed by Styler $\&$ Howie (2014) to measure shear wave velocities along vertical direction of soil specimens [12]. Also bidirectional bender element was used to evaluate anisotropic shear moduli on different planes by Pennington et al. (1997), Ng et al. (2004) and Ng \& Yang (2008) [13-15].

The aforementioned studies and their findings encouraged the authors to investigate the fabric tensor evolution of sands with laboratory tests. The tests are carried out using an automated triaxial apparatus through implanting bender elements in both vertical and horizontal directions. The shear wave velocities (Vs) have been measured in both directions at the end of consolidation as well as during the process of shearing up to critical state.

\footnotetext{
${ }^{*}$ Corresponding author: kfakhari@aut.ac.ir, kfakhari@yahoo.com
} 


\section{TESTING APPARATUS MEASURING DEVICES}

\subsection{Testing apparatus}

In this study, an in-house developed automated triaxial apparatus capable of closed loop control is used in order to perform the testing program. Continuous shear wave velocity measurements in horizontal and vertical directions throughout shearing are monitored. A function generator and an oscilloscope are equipped to send and receive waves. A voltage amplifier is used to properly induce wave amplitudes in order to transcend limitations of function generator in providing high voltage amplitudes of the sine-wave.

In the bender element test, the propagation time of a shear wave through the soil specimen in horizontal and vertical directions is measured simultaneously. Measurement of current tip to tip distances as wave traveling distance in both directions is necessary to determine shear wave velocities. The current vertical traveling distance during shearing is obtained by subtraction the axial deformation (measured by an LVDT) from height of the specimen. However, accurate horizontal wave traveling distance cannot be directly measured during shearing. Thus, a rigid frame is prepared to place a camera in front of triaxial cell to capture the radial deformation of specimen during shearing (Fig. 1). The mentioned snapshots from the specimens are analysed by two image processing software (Ncorr-Open source 2D digital image correlation MATLAB and ImageJ) to identify the failure pattern and horizontal traveling distance. The image processing method is preferred to mounted radial strain gauge, as there are not any local destructive effects involved in operation of horizontal bender elements. A Canon 550D camera with 18 Megapixels APS-C CMOS sensor is featured with two morning glory lamps to eliminate the reflected light by plaxiglass of the cell.

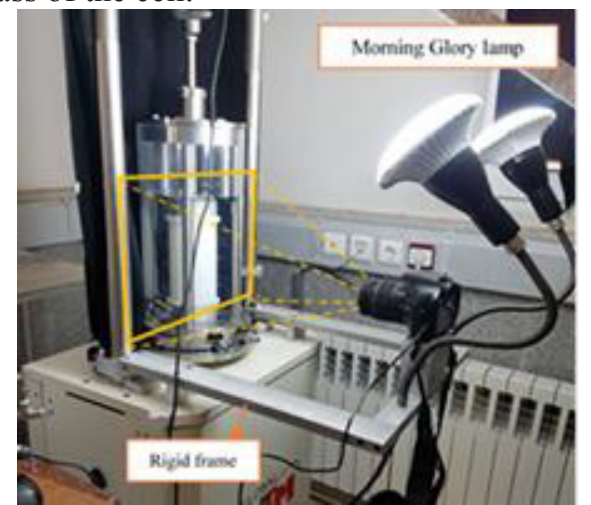

Fig. 1. Image processing frame and two morning glory lamps

\subsection{Image processing verification}

Verification of image processing as a non-destructive method for radial deformation of specimen is performed by three cylindrical Teflon dummies with 60, 70 and 90 $\mathrm{mm}$ in diameter. The mentioned dummies are placed in triaxial cell which is filled by water to show the magnification effect due to the refraction of light in the water and the plaxiglass cell wall (Fig.2). These are split equally into 10 sections in order to evaluate distortion of images along the height of specimens. As shown in Fig.3, there is a clear linear relationship between diameters of the three dummies in images (pixel) versus its real diameter for every section. While, we can observe the curves have deviation from each other along the height of specimen due to perspective distortion which is ignored in this research. As the major purpose of image processing in this research is determination of horizontal wave traveling distance during shearing, therefore the perspective distortion does not have a major effect on the results.

\subsection{Horizontal bender element mounting}

The placement of horizontal bender elements on the sample is a difficult and delicate task. The attachment should be light and with perfect sealing performance. The proposed method in this study is presented in Fig. 4. The assembly includes latex earrings, bender capsules, bender housings, three rotary sealing and several O-rings. The latex earrings are made by dipping an aluminium mold into a liquid latex tank to create an earring on the specimen latex membrane. This latex earring provides a sealant coating for inner assembly of horizontal bender elements. The molds are replaced by bender element capsules using three rotary sealing for bender elements housing to prevent any leakage from the cell chamber to the soil specimen and vice versa. In fact, bender housing plays the role of a shaft for the mentioned rotary sealing. Several O-rings seal effectively the contact of latex earrings and bender element capsules.

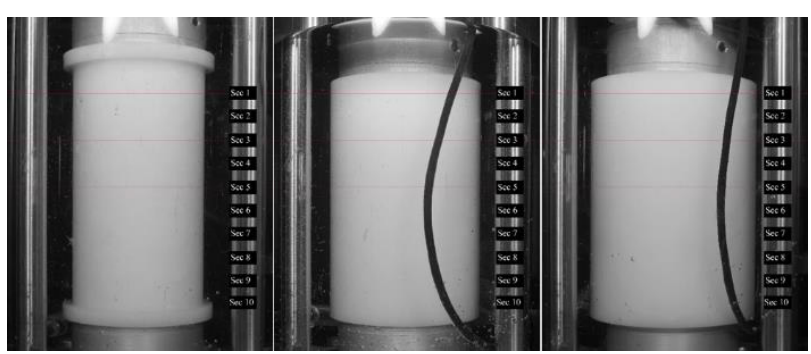

Fig. 2. Placement of cylindrical dummies for considering magnification and distortion effects

\section{MATERIAL PROPERTIES AND SPECIMEN PREPRATION}

\subsection{Material properties}

The soil used in this study is the synthetic Firuzkuh sand F161, which is a gap graded sand. Firuzkuh sand is manufactured industrially with crusher in north-east of Tehran. Gradation analysis of Firuzkuh sand has been performed according to ASTM D 422 standard as presented in Fig. 5. Scanning Electron Microscopy (SEM) of particle placement is presented in the figure for this sand at relative density of 30 percent using the proposed method of Jang et al. (1999) [16]. In this method, low 
viscosity epoxy materials are injected into the soil under controlled conditions. Physical properties of the Firuzkuh sand are briefly shown in Table 1 . The gradation curve and a magnified image of the particle shapes are shown in Fig. 7.

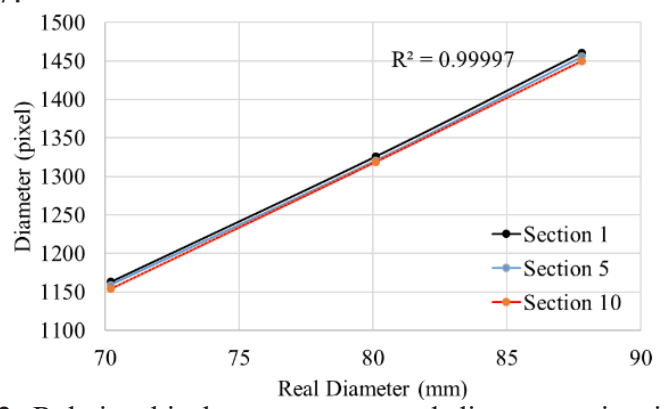

Fig. 3. Relationship between measured diameter using image processing method VS directly measured diameter

\subsection{Specimen preparation}

In addition, both drained and undrained monotonic triaxial tests were conducted. The diameter and height of samples are $71 \mathrm{~mm}$ and $160 \mathrm{~mm}$, respectively. The samples were prepared by Wet Tamping method. The horizontal bender elements during sample preparation are located in parking spot (bender capsule) as shown in Fig. 4 and after applying the internal suction, the bender element housings were inserted gently into the soil specimen. In addition, the bender element probes are premounted in the upper plate and the pedestal for vertical wave propagations.

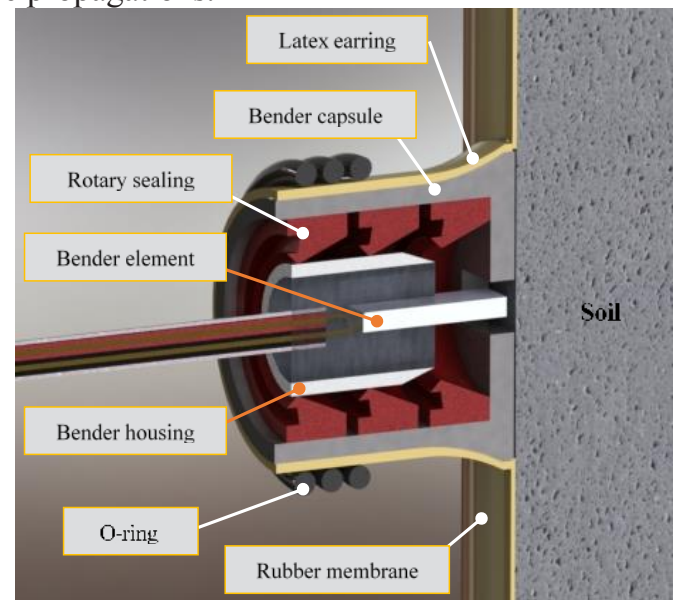

Fig. 4. Cross section of horizontal bender element assembly

In order to carry out the specimen saturation process, carbon dioxide gas and de-aired water were percolated through the specimen, while the confining pressure of 20 $\mathrm{kPa}$ was applied to the specimen. The backpressure was used to remove small air bubbles in the specimen and avoid the cavitation phenomenon under low pore pressure. Strain-controlled shearing of specimens is performed after the isotopic consolidation. Measurement of shear wave velocity in vertical and horizontal directions is scheduled to commence at the end of consolidation as well as during shearing.
Testing program is displayed in Table 2 . The void ratio and relative density at the end of consolidation are presented in the table. The void ratio at the end of the test was achieved by the proposed method of Verdugo \& Ishihara (1996) [18].

Table 1. Physical properties of Firuzkuh sand

\begin{tabular}{|c|c|c|c|c|c|}
\hline $\mathrm{C}_{\mathrm{c}}$ & $\mathrm{C}_{\mathrm{u}}$ & $\mathrm{D}_{50}(\mathrm{~mm})$ & $\mathrm{G}_{\mathrm{s}}$ & $\mathrm{e}_{\max }$ & $\mathrm{e}_{\min }$ \\
\hline 0.88 & 1.9 & 0.22 & 2.65 & 0.88 & 0.54 \\
\hline
\end{tabular}
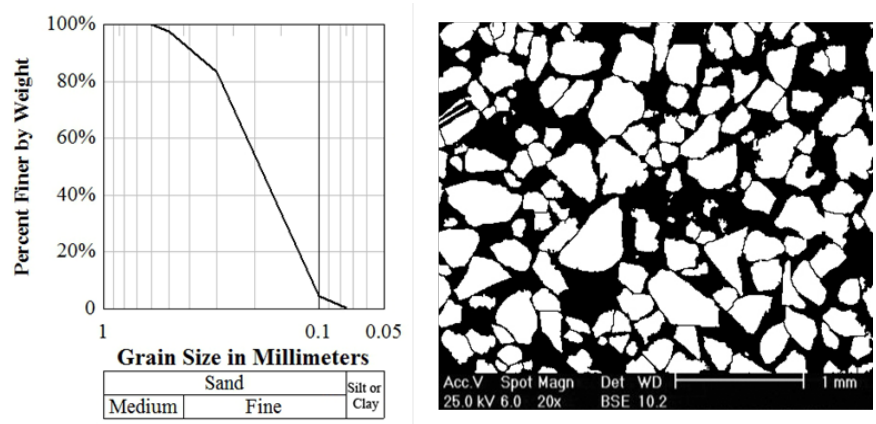

Fig. 5. Gradation of Firuzkuh F161 sand and scanning electron microscope of particle placement

Table 2. Outline of the testing program

\begin{tabular}{|c|c|c|c|c|}
\hline Test name & $\mathrm{p}_{\mathrm{c}}(\mathrm{kPa})$ & $\mathrm{e}$ & $\mathrm{D}_{\mathrm{r}}(\%)$ & Drainage \\
\hline TD13PD100 & 100 & 0.882 & 13 & Drained \\
\hline TD16PD100 & 100 & 0.873 & 16 & Drained \\
\hline TD25PD100 & 100 & 0.84 & 25 & Drained \\
\hline TD13PU100 & 100 & 0.882 & 13 & Undrained \\
\hline TD16PU100 & 100 & 0.873 & 16 & Undrained \\
\hline TD26PU100 & 100 & 0.839 & 26 & Undrained \\
\hline
\end{tabular}

\section{TEST RESULTS}

In order to clarify the role of relative density on the behaviour of drained specimens, the stress paths (q-p'), deviatoric stress versus axial strain and e-p' curves for different specimens are illustrated in Figs. 6 through 8, respectively. The critical state line (CSL) and Phase Transformation Line (PTL) in e-p' space which has been obtained from previous studies on Firuzkuh sand using triaxial apparatus are illustrated in order to examine the initial conditions of specimens at e-p' space with respect to CSL and PTL, as explained by Yoshimine and Ishihara (1998). The e and p' denote the void ratio and mean effective stress, respectively. As it can be seen in Fig. 7, the deviatoric stresses are raised as the relative densities are increased. But all drained specimens at the end of shearing have approached the same deviatoric stress, known as critical state. This tendency to reach the critical state occurs for drained specimens at the end of shearing as presented in e-p' space of Fig. 8.

\subsection{Stress-strain and volume change behaviour}

Figure 6 shows the effective stress paths for drained and undrained specimens. The specimens were prepared with

\subsection{Testing program}


different relative densities and consolidated under mean effective stress of $100 \mathrm{kPa}$. The effects of variations of relative density and drainage condition on the behaviour of specimens are observed in Fig. 6. It is noticed that Firuzkuh sand is very sensitive to initial relative density as under Dr of 13 and $16 \%$, the response is entirely contractive, while under a Dr of $25 \%$, the response has shifted to contractive/dilative. This is attributed to high angularity of the crushed particles as shown in Fig. 5.

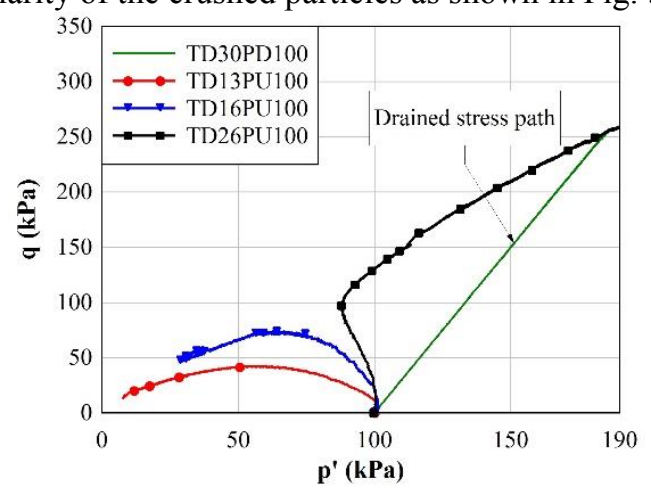

Fig. 6. Effective stress paths for all specimens in q-p' space

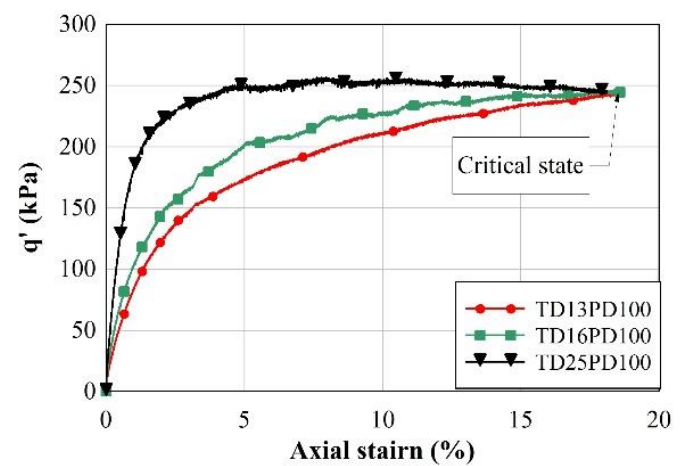

Fig. 7. Deviatoric stress vs axial strain for drained specimens

\subsection{Signal Interpretation}

The trend of travel time for both horizontal and vertical directions during shearing were tracked and interpreted in the same manner. Fig. 9 shows an example of signal interpretation of the received and transmitted waves which was performed on TD25PD100 specimen in horizontal direction. The first and the last received signals are related to the end of consolidation (EOC) and the critical state, respectively, and the other waves are captured during the specimen shearing. In addition, the stress states for all specimens during shearing are presented in Figs. 12 and 13. It is observed that the wave form of the received signals during shearing are almost similar and only some small translation in the received times causes the variations of travel time. The travel time of the specimen decreases progressively and then increases. The shear wave velocity is determined by dividing travel time by travel distance, although travel distance can be altered based on the failure mode.

In this study, the horizontal and vertical travel distances were measured using the image processing method and an axial LVDT, respectively. In Fig. 10, a snapshot from the results of image processing of one specimen are presented indicating the horizontal and vertical displacement contours. The general pattern of failure for this specimen is bulging in which the capsules of horizontal benders moved away from each other. On the other hand, the amplitude of received signals gradually decreased because of an increase in the travel distances.

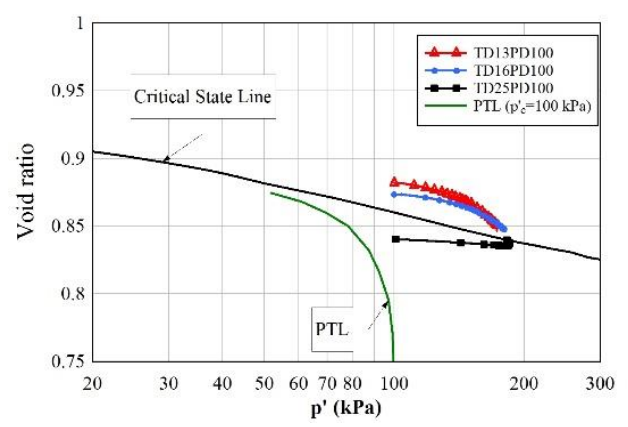

Fig. 8 Variations of void ratio for drained specimens

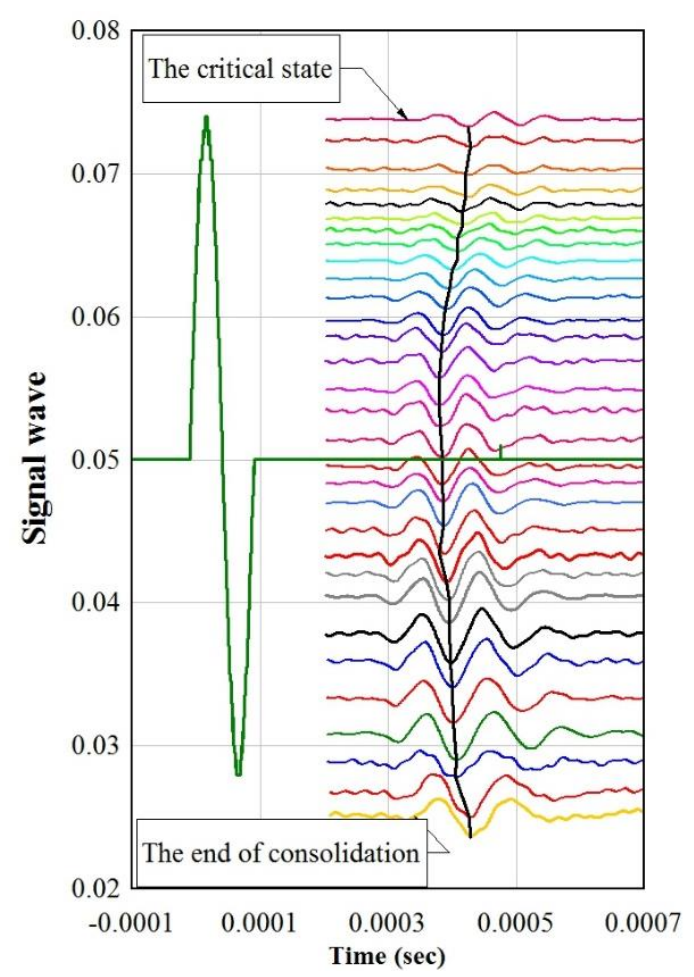

Fig. 9. Trend of travel time of horizontal direction of TD25PD100 specimen from EOC to critical state

In order to determine the travel distances during shearing, the ImageJ software was used to analyse the taken snapshots. The border of the specimen was defined by the clear profile against the dark background as shown in Fig. 11. Two target points were marked by permanent ink at latex earrings. Furthermore, the distance between the two mentioned target points were accurately measured after applying internal suction for additional verification. The travel distance in every snapshot was defined by considering the variation of specimens' radius on initial tip to tip bender distance.

\subsection{Shear wave velocities}

The vertical and horizontal shear wave velocities for drained specimens are illustrated in Fig. 12 in which Vs ${ }^{\text {vh }}$ 
and $\mathrm{Vs}^{\text {hv }}$ are the shear wave velocity in vertical and horizontal direction, respectively.

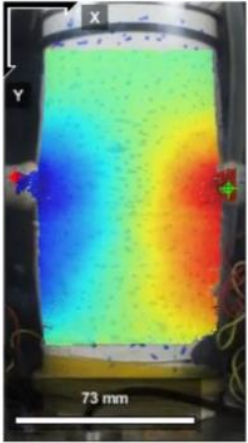

Horizontal

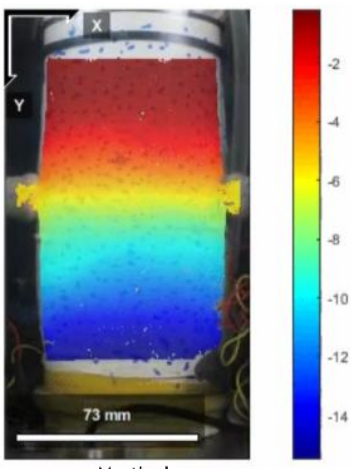

Vertical
Fig. 10. The horizontal and vertical displacement contours

The first and second superscripts denote the direction of wave propagation and wave polarization, respectively. The deviatoric stress and void ratio variations due to shearing are presented as a second independent $\mathrm{Y}$ axis for better understanding and ease of comparisons. As expected, specimens with greater relative density had larger shear wave velocity at the end of consolidation due to a stronger interlocking of grains.

The shear wave velocities of all specimens initially increased as loading commenced and then eventually converged to a unique state in both horizontal and vertical directions. As shown in the figure, the steady state of swave velocity for all the specimens is about $177 \mathrm{~m} / \mathrm{s}$ for all the specimens consolidated at mean effective stress of $100 \mathrm{kPa}$.
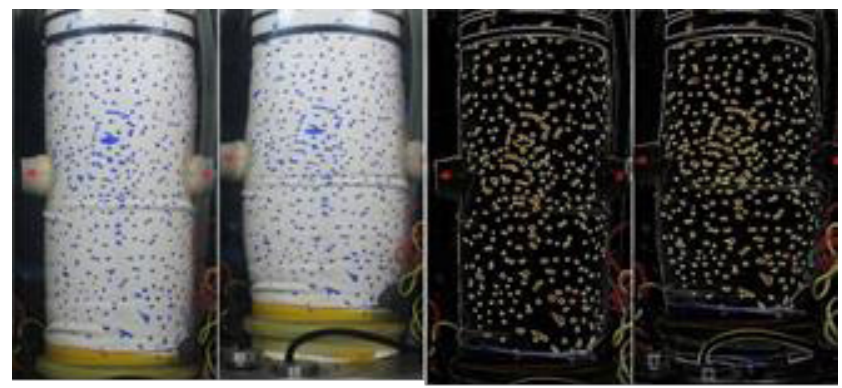

Fig. 11. Locating the boarder of specimens during shearing

Similarly, the shear wave velocities of undrained specimens for both horizontal and vertical directions are presented in Fig. 13. The excess pore water pressure and deviatoric stress curves versus axial strain for mentioned specimens are illustrated as second $\mathrm{Y}$ axis for further clarifications. The stiffness of loose specimens in both directions initially decrease due to the rising of the excess pore water pressure and then gradually approach a unique shear wave velocity like the drained specimens, the magnitudes of which are equal to the corresponding drained specimens. On other hand, the stiffness of TD25PU100 specimen initially increased and then smoothly decreased to the same unique shear wave velocity.

It seems that the global stiffness of all the examined specimens at the critical state has an isotropic condition, i.e. identical shear wave velocity (and hence modulus) are obtained. This is while the previous studies had shown that there is an intense anisotropic fabric at critical state. It is noticeable that shear wave velocity is a function of stress state and fabric in which fabric is characterized by grains shape, minerals, arrangement, number and type of inter-granular contacts. Therefore, the shear wave velocity is not simply represented by fabric conditions.
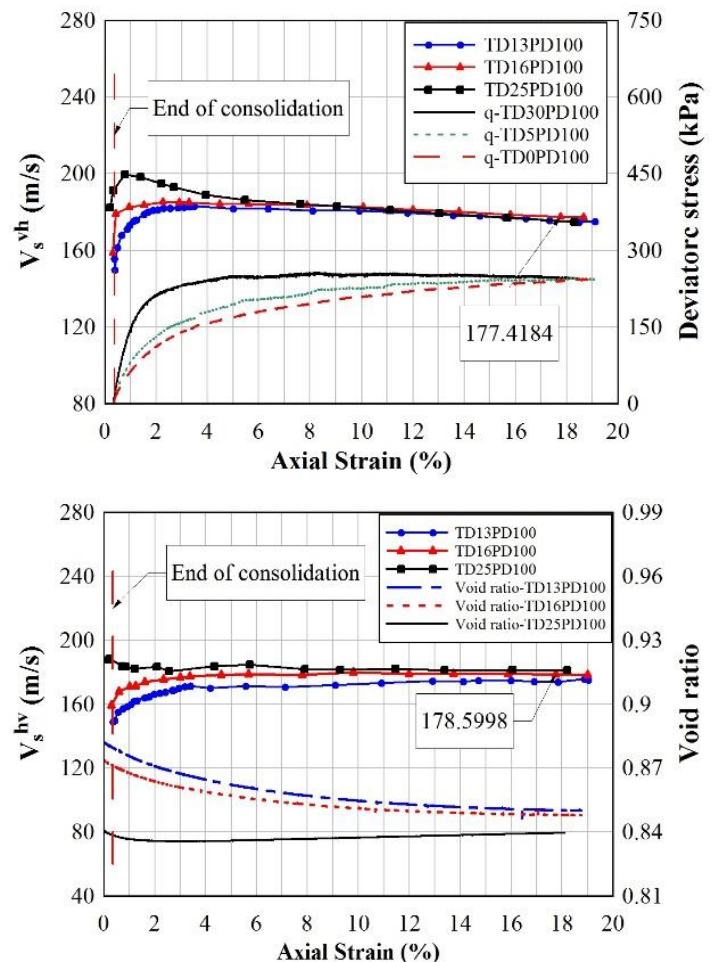

Fig. 12. Vertical and horizontal shear wave velocities for drained specimens
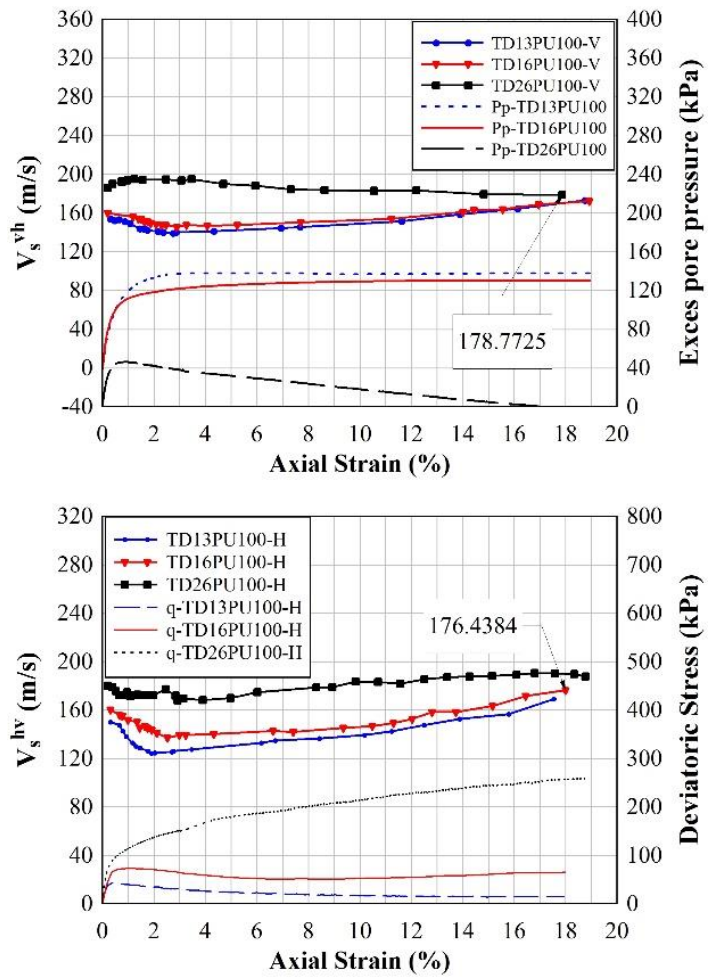

Fig. 13. Vertical and horizontal shear wave velocities for undrained specimens 
Stress states in horizontal and vertical directions are not identical during shearing and at critical state. Hence the shear wave velocity in the mentioned directions cannot necessarily be considered as an index of fabric conditions.

Moreover, there are some variations of local void ratio in specimens due to failure mode at critical state, while shear waves should pass through non-homogeneous paths. But at critical state along a specific direction, as the stress state continues to remain constant, so the shear wave velocity can be represented as a fabric index. Thus, the fabric condition at critical state in both directions approached a specific state which was observed in previous studies of Li \& Dafalias (2012) and Yan \& Zhang (2013) by DEM analyses and characterized experimentally in this study. More studies and experiments are underway to further clarify this significant issue.

\section{CONCLUSIONS}

The possibility of experimentally capturing the fabric evolution during shearing was exercised by bidirectional shear wave velocity measurements in which two horizontal piezo-electrics were mounted on samples using a new measurement technique. This was made feasible by three rotary seals which were implanted in bender capsules. Three drained and three undrained traixal compression tests were performed on the crushed Firuzkuh silica sand. In order to determine the travel distance of the wave propagation along horizontal direction, image processing method was used. The main findings of this work are twofold: (1) it is observed that shear wave velocity measurement in two horizontal and vertical directions could be useful to characterize the fabric evolution, and (2) the fabric condition of examined specimens at critical state are constant. This issue has also been represented by DEM simulations (Li \& Dafalias, 2012and Yan \& Zhang, 2013) which is examined in this study experimentally.

More studies are required however, to further evaluate the effects of different confining pressure, initial void ratio, stress paths, etc., on the fabric characteristics during the process of shearing.

The authors would like to acknowledge the contributions of Global Material Testing Manufacturers (Global MTM) for their sincere supports and cooperation over equipment setup and accessories development to enable the testing program of this study.

\section{References}

1. Roscoe, K. H_A. Schofield, and C. P. Wroth. "On the yielding of soils." Geotechnique 8, no. 1 (1958): 2253.

2. Schofield, A., \& Wroth, P. (1968). Critical state soil mechanics (Vol. 310). London: McGraw-Hill.

3. Nakata, Yukio, Masayuki Hyodo, Hidekazu Murata, and Noriyuki Yasufuku. "Flow deformation of sands subjected to principal stress rotation." Soils and Foundations 38, no. 2 (1998): 115-128.

4. Yoshimine, Mitsutoshi, and Kenji Ishihara. "Flow potential of sand during liquefaction." Soils and Foundations 38, no. 3 (1998): 189-198.

5. Oda, Masanobu. "The mechanism of fabric changes during compressional deformation of sand." Soils and foundations12, no. 2 (1972): 1-18.

6. Masson, S., and J. Martinez. "Micromechanical analysis of the shear behaviour of a granular material." Journal of engineering mechanics 127, no. 10 (2001): 1007-1016.

7. Li, Xia, and Xiang-Song Li. "Micro-macro quantification of the internal structure of granular materials." Journal of engineering mechanics 135, no. 7 (2009): 641-656.

8. Li, Xiang Song, and Yannis F. Dafalias. "Anisotropic critical state theory: role of fabric." Journal of Engineering Mechanics138, no. 3 (2011): 263-275.

9. Yan, W. M., and Lin Zhang. "Fabric and the critical state of idealized granular assemblages subject to biaxial shear." Computers and Geotechnics 49 (2013): 43-52.

10.Sadrekarimi, A., and S. M. Olson. "Critical state friction angle of sands." Géotechnique 61, no. 9 (2011): 771.

11. Shirley, Donald J., and Loyd D. Hampton. "Shearwave measurements in laboratory sediments." The Journal of the Acoustical Society of America 63, no. 2 (1978): 607-613.

12.Styler, Mark A., and John A. Howie. "Continuous monitoring of bender element shear wave velocities during triaxial testing." Geotechnical Testing Journal 37, no. 2 (2014): 218-229.

13.Pennington, D. S., D. F. T. Nash, and M. L. Lings. "Anisotropy of G0 shear stiffness in Gault Clay." Géotechnique 47, no. 3 (1997): 391-398.

14.Ng, Charles WW, Erin HY Leung, and C. K. Lau. "Inherent anisotropic stiffness of weathered geomaterial and its influence on ground deformations around deep excavations." Canadian Geotechnical Journal 41, no. 1 (2004): 12-24.

15.Ng, Charles Wang Wai, and S. Y. Yung. "Determination of the anisotropic shear stiffness of an unsaturated decomposed soil." Géotechnique 58, no. 1 (2008): 23-35.

16.Jang, Deh-Jeng, J. David Frost, and Jin-Young Park. "Preparation of epoxy impregnated sand coupons for image analysis." Geotechnical Testing Journal 22, no. 2 (1999): 153-164.

17. Verdugo, Ramon, and Kenji Ishihara. "The steady state of sandy soils." Soils and foundations 36 , no. 2 (1996): 81-91. 